\title{
Minimizing Sum Distortion for Static and Mobile Fusion Center Placement in Underwater Sensor Networks
}

\author{
Satish Vedantam, Urbashi Mitra, Gaurav S. Sukhatme and Filippo Arrichiello \\ University of Southern California and Università degli Studi di Cassino \\ \{vedantam,ubli,gaurav\}@usc.edu and f.arrichiello@unicas.it
}

\begin{abstract}
The problem of optimizing the position of a mobile fusion center in a sensor network is considered. The optimization criterion of interest is the sum distortion for the communication of all the information from each of the nodes to the fusion center. Transmission losses along underwater links are modeled and a time-multiplexing architecture imposed on the sensor nodes for communication with the fusion center. Classical results from the information theory literature are leveraged and the optimization problem is formulated and solved analytically for the case when the fusion center is at one fixed location and numerically for the case when the fusion center is mobile. It is observed that in the low node power regime, with the fusion center at a fixed location, the fusion center selectively communicates with a few nodes while turning the others off. In the high power regime, the time allocated to the nodes is a function of the information they need to transmit to the destination and the distance from the node to the fusion center. Further, when the fusion center is mobile, in the low power regime, the fusion center is placed close to the node with the largest information content while for higher powers the difference from a fixed fusion center declines.
\end{abstract}

\section{INTRODUCTION}

There is interest in the deployment of underwater robotic teams for a number of key applications: e.g. mapping, exploration and monitoring of marine environments, measurement collection for oceanographic missions [1], autonomous navigation in formation [2] or autonomous operations in harbors; moreover, they can be used as nodes of mobile underwater sensor networks [3]. Herein, we examine a heterogeneous network comprised of static sensing nodes as well as robotic nodes which can behave as data mules or mobile fusion centers. To this end, we are currently instrumenting robotic boats to have underwater acoustic communications capabilities. These robotic boats are an integral part of the Networked Aquatic Microbial Observing System (NAMOS) project ${ }^{1}$, which is used in studies of microbial communities in freshwater and marine environments [4], [5]. The NAMOS testbed is a system

U. Mitra and S. Vedantam are supported in part by the following grants and organizations: NSF OCE-0520324, NSF CNS-0722073, NSF CNS-0821750 (MRI), and the University of Southern California's Provost Office.

G. Sukhatme is supported in part by the NSF under grants CCR0120778 and CNS-0540420; the NOAA MERHAB program under grant NA05NOS4781228 and the ONR MURI program under grant N00014-081-0693.

${ }^{1}$ See http://robotics.usc.edu/ $/$ namos for more information.

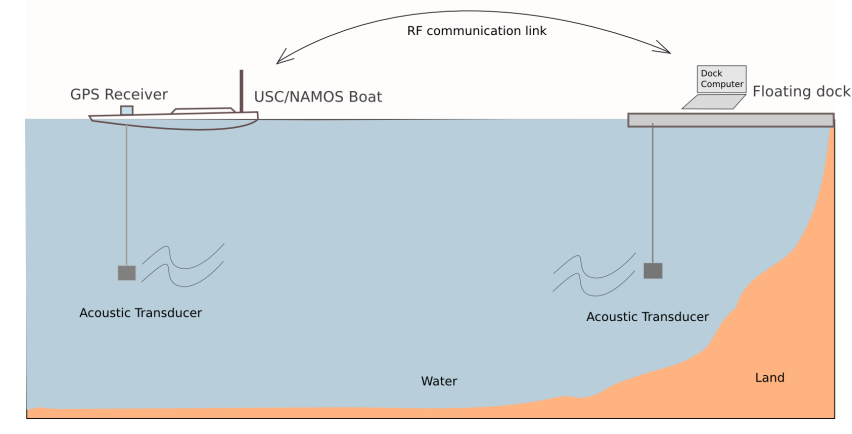

Fig. 1. Setup for testing and benchmarking the NAMOS system.

of anchored buoys (the static nodes), and a robotic boat (the mobile robot) capable of measuring temperature and chlorophyll concentrations. Thus, the testbed is a collection of static nodes as well as mobile/actuated nodes. A key research goal of the NAMOS project is determining how to coordinate the mobile robots and the static nodes such that the error associated with the estimation of a scalar field is minimized subject to the constraint that the energy available to the mobile robot is bounded. Specifically, if each static node makes a measurement, and the total energy available to the network is known, what path should the mobile robot take to minimize the integrated mean square error (MSE) associated with the reconstruction of the entire field? Typically, it had been assumed that the energy consumed by communication and sensing was negligible compared to the energy consumed by moving the mobile robot [6]. Another common assumption was that the mobile robot could communicate essentially errorfree with all the static nodes and acquire sensor readings from them. In the currently implemented NAMOS system, communication is over a multi-hop wireless ad-hoc network described in [4]. This network employs 802.11 and thus communicates over-the-air. With the inclusion of underwater acoustic communication, we will have a proxy for a truly underwater mobile robot such as a glider or small submarine. In this new context, the cost of communications and the variability of the communication channel cannot be ignored.

Thus, in addition to examining mobile node location as a function of estimation accuracy, we shall also consider the 
impact of endeavoring to communicate as well as estimate over the channel. We shall consider only underwater acoustic channels in this work. The problem of communicating over a link while estimating the parameters associated with the link is addressed in [7]. There, it is established that a distortion constraint on the quality of the channel estimates at the destination node can be translated to a constraint on the codebook employed at the encoder under some mild constraints. The problem is also generalized to the Multiple Access Channel (MAC) and the two-hop network, where, we are interested in estimates for all the channels involved in the network. The results of that work are then directly applicable to this problem when we are interested in forming channel estimates in addition to communicating over the network.

In the sensor networking community, adapative sampling techniques have been used to address the tradeoff between network lifetime and estimation accuracy. In the typical setting the network is used to estimate a field by means of extrapolation from samples - measurements made at the locations where the network nodes are deployed. Approaches to treat this trade-off vary from multi-scale wavelet-based techniques [8] to linear filtering for sampling rate adaptation [9]. In the traditional paradigm, energy expenditure in a sensor network is largely ascribed to communication so prolonging network lifetime is often equated with switching off the radios on selected sensor nodes. If the sensor network is heterogeneous, and some nodes within it are mobile, then the energy expenditure is largely due to mobility. Rahimi [10] proposed an algorithm for an actuated sensor network, which successively refines the field estimation by taking more readings in locations where estimation variance is high. In [11] a similar approach (based on optimal experimental design) is used, but a specific model of energy consumption due to mobility is used. The approach in [12] assumes that many sensors are deployed at the beginning; the correlations between the values of the scalar field at different locations are learned from the data collected from the sensors and the sensors are then redeployed for optimal sampling. In the mobile robotics community, the related problem of efficient data gathering tours by mobile robots has received some attention. The setting is usually in the context of accurate self-localization by the robot [13], or simultaneous self-localization and environment mapping [14] or efficient environment coverage via mobility [15]. In the theoretical computer science community, similar problems have been studied as variants of TSP [16] and [17].

In contrast, this work draws heavily from the results from classical estimation theory (see [18] for example). We also employ classical results in the information theory community (see [19], for example) to solve the minimum sum distortion problem when we have multiple sensors and a single fusion center. The results of this optimization are then extended to the case when the fusion center is mobile and results are derived by simulation. Despite the fact that our paper relies quite heavily on classical results in the literature, we use these results in a novel fashion to solve a fundamental problem for sensor networks. We will assume no fading for the remainder of this paper. Further, we will make simplifying assumptions to drive home the tradeoffs that we want to highlight. This will help us generate baseline estimates for the optimizing parameters which can then be improved by employing the applicable fading models. Finally, we assume a unit bandwidth for each link, for the purposes of this paper since we are concerned with the optimal location of the relay. The problem under the availability of a band of frequencies can be addressed using the tools presented herein via appropriate scaling of the rates on the corresponding links. One particular feature of the underwater channel is the decay in available bandwidth with increasing inter-node distances. This particular issue will be addressed at a later stage.

We impose a time multiplexed communication scheme between the fusion center and each of the sensing nodes for the communication of estimates. It is observed that for low node SNR's, it is optimal to selectively turn off some nodes in the network - the fusion center. Conversely, at high SNR's for the sensing nodes, the time allocated to each sensor is a function of its distance from the fusion center and the "amount" of information that it has for the fusion center. These results are similar to those presented in [20]. Further, there are gains from optimizing for the location of the mobile sensor which increase as the size of the network grows from the few hundred metres in this work to the larger distances for more practical real-world networks. These gains are also evident at higher operating frequencies since the losses in underwater communication grow large in this regime.

The remainder of this paper is organized as follows: Section II describes the setup used to collect the data analyzed in this paper and presents some statistics on the data collected, Section III presents an overview of the challenges presented by underwater acoustic communication, Section IV presents an introduction to information theory for the purposes of this paper, Section $\mathrm{V}$ presents analysis and results for the case when the buoys communicate their information to a fixed fusion center, Section VI presents analysis for the case when the fusion center can be moved to further minimize distortion and finally, Section VII concludes this paper with some ideas for future work.

\section{DAta Collection}

\section{A. Setup}

The data used for this paper was collected at James Reserve (Lake Fulmor), CA from August 28th to September 1st, 2006. Nine buoys were deployed in the lake and extensive amounts of data was collected over the time frame of the experiment. Some raw results are available on the NAMOS webpage ${ }^{2}$. We will be using only a section of the data collected. In particular, we are interested in the surface temperature recorded at each of the buoys. Since surface temperature varies from buoy to buoy, we note that each buoy has information to transmit to the fusion center.

\section{B. Collected Data}

Table I presents the surface temperature at each of the buoys and their locations for the network presented in Figure 2. Note

\footnotetext{
${ }^{2}$ See http://robotics.usc.edu/rnamos/data
} 


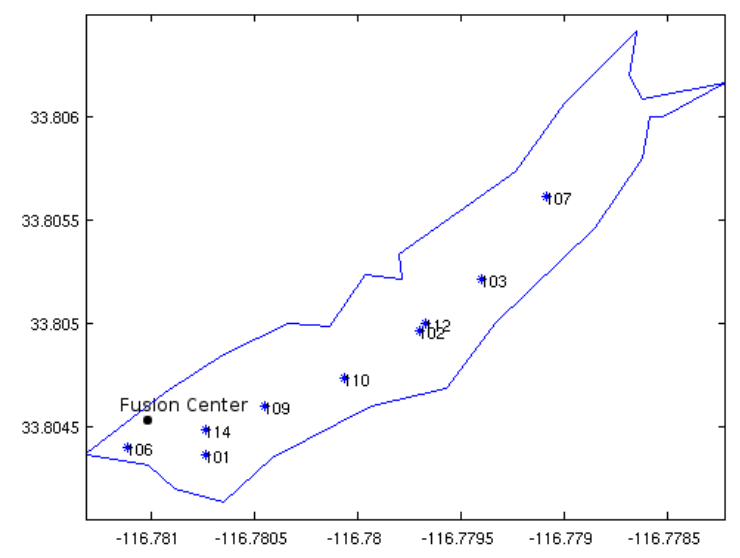

Fig. 2. Buoy setup to collect data.

TABLE I

STATISTICS FOR THE DATA COLLECTED FROM THE NETWORK PRESENTED

IN FIGURE 2.

\begin{tabular}{|c|c|c|c|c|}
\hline $\begin{array}{c}\text { Node } \\
\text { Number }\end{array}$ & $\begin{array}{c}\text { Longitude } \\
{ }^{\circ} \mathrm{W}\end{array}$ & $\begin{array}{c}\text { Latitude } \\
{ }^{\circ} \mathrm{N}\end{array}$ & $\begin{array}{c}\text { Mean } \\
{ }^{\circ} \mathrm{C}\end{array}$ & Variance \\
\hline \hline 101 & -116.780733 & 33.804367 & 20.1285 & 0.8016 \\
102 & -116.779700 & 33.804967 & 19.8805 & 1.8452 \\
103 & -116.779400 & 33.805217 & 19.5718 & 1.9775 \\
106 & -116.781117 & 33.804400 & 33.6061 & 118.6918 \\
107 & -116.779083 & 33.805617 & 19.4780 & 1.9810 \\
109 & -116.780450 & 33.804600 & 19.9139 & 1.1469 \\
110 & -116.780067 & 33.804733 & 19.9810 & 1.5435 \\
112 & -116.779667 & 33.805000 & 19.8571 & 1.7236 \\
114 & -116.780733 & 33.804483 & 19.7265 & 1.0698 \\
\hline
\end{tabular}

that the data from Node 106 seems to have an especially high mean and variance (approximately 33 and 118 respectively) leading one to suspect that the measurements on that node are bad. So, we will refrain from using the data from this node for the remainder of this paper and concentrate on the eight nodes remaining as the sources of interest.

\section{UNDERWATER COMMUNICATION}

Characterizing the underwater acoustic channel has been a subject of great interest in the literature the past decades [21], [22]. As pointed out in [23], there exists fundamental differences that distinguish underwater acoustic channel from a Radio Frequency (RF) land mobile channel or a satellite channel. As unique to underwater acoustic channels, the signal frequency plays an important role of determining the absorption loss due to transfer of acoustic energy into heat. Relying on extensive laboratory and field experiment data, the empirical formula of the attenuation coefficient $\beta$ with units of $\mathrm{db} / \mathrm{km}$ in sea water is presented in [24] and shown in Equation (1), where $f$ is frequency in $\mathrm{kHz}, A=2.34 \times 10^{-6}$, $B=3.38 \times 10^{-6}, S$ is salinity, $P$ is hydrostatic pressure in $\mathrm{kg} / \mathrm{cm}^{2}$, and

$$
f_{T}=21.9 \times 10^{6-\frac{1520}{T+273}},
$$

is a relaxation frequency in $\mathrm{kHz}$, with $\mathrm{T}$ the temperature in ${ }^{\circ}$ C. $f_{T}$ fluctuates between $59 \mathrm{kHz}$ and $210 \mathrm{kHz}$ as temperature ranges from $0^{\circ} \mathrm{C}$ to $30^{\circ} \mathrm{C}$. For lower range of frequencies (i.e. $100 \mathrm{~Hz}$ to $3 \mathrm{kHz}$ ), the empirical formula for $\beta$ is also known as Thorp's formula. Once the attenuation coefficient is determined, the transmission loss can be evaluated by

$$
20 \log _{10}\left(\frac{\mathrm{SNR}_{d}}{\mathrm{SNR}_{0}}\right)=-\beta d
$$

where, $\mathrm{SNR}_{0}$ is the Signal to Noise Ratio at the transmitter and $\mathrm{SNR}_{d}$ is that at a distance of $d \mathrm{~km}$. The transmission loss due to sound absorption leads to a fundamental bandwidth limitation of the underwater acoustic channel. The other characteristics of the underwater acoustic channel are described in greater detail in [23], [21], [22]. These features include timevarying channels due to Doppler effects, large delay spreads due to sparse multipath and significant latencies due to the slow speed of sound in water. However, for the current work, we shall focus on the strong attenuation of underwater acoustic propagation as a function of distance.

\section{A REVIEW OF RELEVANT INFORMATION THEORY CONCEPTS}

In this section, we present a background on information theory as is relevant to this paper. We will deal with discrete random variables for ease of exposition.

Since we already have observations from each of the buoys in our network, we are primarily concerned with compressing the information at each node and communicating it over a noisy channel to the data sink. This simple construct invokes the two fundamental classes of problems in information theory: source coding and channel coding. Source coding is the process of compressing observations from a random variable to the minimum information possible while channel coding is the process of introducing redundancy in a signal to ensure perfect reconstruction at the destination node.

Theorem 1 (From [25]): With every given discrete memoryless channel, we associate a constant $C$ such that: for any $\epsilon>0$ and and communication rate $R<C$, for large enough $N$, there exists a block code of length $N$ and rate $\geq R$, and a decoding algorithm, such that the maximal probability of block error is $<\epsilon$. Further, if $R>C$, as $N \rightarrow \infty, \mathrm{P}$ (error) $\rightarrow 1$.

Theorem 2 (From [25]): A source that produced independent and identically distributed random variables with entropy $H$ can be encoded with arbitrarily small error probability at any rate $R$ (in bits per source output) if $R \geq H$. Conversely, if $R<H$, the error probability will be bounded away from zero, independent of the complexity of coder and decoder.

These are the famed Noisy Channel Coding and the Source Coding theorems (and their converses) first proved by Shannon. They define the constraints we need to meet to have sustained error-free communication for any communication problem in general and our problem in particular.

We now discuss some information theoretic results that are relevant to our current problem. First, consider the general network presented in Figure 3. Here, we have $M$ sources of information trying to communicate their data to the destination. If the destination is interested in perfect reconstructions for the data from each of the nodes, the capacity region for this problem is given by the solution of the Multiple Access 


$$
\beta= \begin{cases}\frac{0.11 f^{2}}{1+f^{2}}+\frac{44 f^{2}}{4100+f^{2}} & f \in(100 \mathrm{~Hz}, 3 \mathrm{kHz}] \\ 8.68 \times 10^{3}\left(\frac{S A f_{T} f^{2}}{f_{T}{ }^{2}+f^{2}}+\frac{B f^{2}}{f_{T}}\right)\left(1-6.54 \times 10^{-4} P\right) & f \in(3 \mathrm{kHz}, 0.5 \mathrm{MHz}]\end{cases}
$$

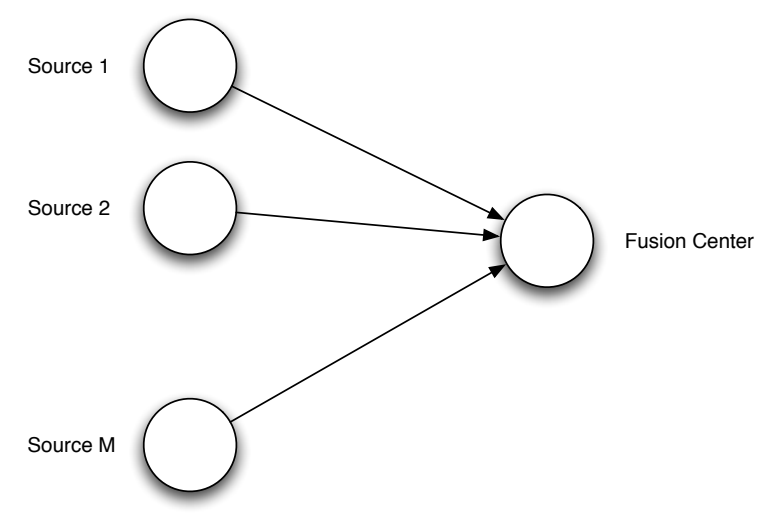

Fig. 3. The many-to-one network.

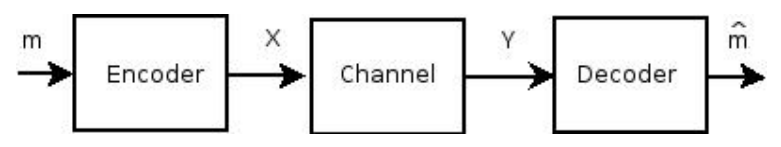

Fig. 4. The two node network.

Channel (since there is a common medium that is used to communicate all the messages from all the nodes) - see [19] for a solution.

However, if the destination were to be interested in a (possibly) distorted version of the data from only one of the nodes the problem changes considerably. The problem is now called the Many-Help-One problem and is unsolved in general (see [26] for a deeper discussion.) The problem we are interested is one where the destination is interested in (possibly) distorted versions of the data from each of the nodes. This is a considerably harder problem to address and is another open problem in general. We will hence take a Time Domain Multiple Access (TDMA) view to present an achievable sum distortion rate for the problem.

We will employ a few other results from the information theory literature which we elaborate on now. Consider the source-channel-destination link presented in Figure 4. If $X \sim$ $\mathcal{N}(0, P)$ and the channel is an Additive White Gaussian Channel (AWGN), the output of the channel is given by:

$$
Y=X+Z
$$

where, $Z \sim \mathcal{N}(0, N)$. Further, if both $X$ and $Z$ are assumed to be independent and identically distributed (i.i.d), the capacity of this channel is given by

$$
C=\frac{1}{2} \log \left(1+\frac{P}{N}\right)
$$

as established in [25].

Further, given an i.i.d Gaussian source, $X \sim \mathcal{N}\left(0, \sigma^{2}\right)$, if we are willing to tolerate a distortion threshold of $D$, assuming

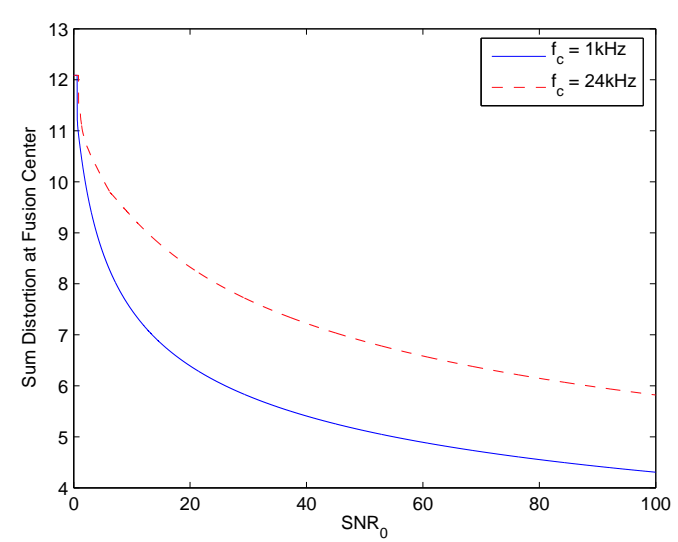

Fig. 5. Sum distortion at the fusion center as a function of the initial transmit SNR at each of the buoys for $f_{c}=1 \mathrm{kHz}$ and $f_{c}=24 \mathrm{kHz}$.

a mean squared distortion metric:

$$
d(X, \hat{X})=\mathbb{E}\|X-\hat{X}\|^{2}
$$

then the minimum rate that the channel in Figure 4 needs to sustain is given by the rate-distortion function:

$$
R(D)=\frac{1}{2} \log \left(\frac{\sigma^{2}}{D}\right)
$$

Again, the proof for this result can be found in [19].

\section{Problem Setup With Static Fusion Center}

We will model the data collected at each of the sources by an i.i.d Gaussian source with mean and variance as given in Table I: $X_{i} \sim \mathcal{N}\left(m_{i}, \sigma_{i}^{2}\right)$. Since the amount of data that each node in our network needs to communicate to the fusion center is relatively small, we will assume that the lower frequencies are used for communication between the nodes and the fusion center and so use Thorp's equation (the first branch in Equation (1). Let us assume a centre frequency of $1 \mathrm{kHz}$ for communication. Equation (1) then yields $\beta \sim 44$. However, if we are interested in smaller form-factor devices, we can attempt to use a higher frequency like $24 \mathrm{kHz}$ and then $\beta \sim 124$. Let us model each of the links from the buoys to the fusion center as an AWGN channel with an SNR of $\mathrm{SNR}_{0}$ at each of the buoys. Given the $\beta$ we just calculated, we can then evaluate the corresponding communication rate $R_{i}$ for the link between buoy $i$ and the fusion center:

$$
R_{i}=\frac{1}{2}\left(1+\mathrm{SNR}_{0} 10^{-\frac{\beta d}{20}}\right)
$$

Let $T_{i}$ be the fraction of time that we allocate to node $i$ in the TDMA setting. Then, the effective rate that node $i$ can communicate information to the fusion center is given by $R_{i} T_{i}$. Given this rate of communication between node $i$ 
and the fusion center, if we assume a mean squared distortion metric, the minimum distortion that we can sustain in the estimation of $X_{i}$ is then given by

$$
D_{i}=\sigma_{i}^{2} e^{-2 R_{i} T_{i}}
$$

by Equation (6). The minimum sum distortion problem can then be formulated as

$$
\begin{array}{ll}
\text { Minimize } & D=\sum_{i=1}^{n} D_{i}=\sum_{i=1}^{n} \sigma_{i}^{2} e^{-2 R_{i} T_{i}} \\
\text { subject to } & \sum_{i=1}^{n} T_{i} \leq 1 \quad \text { and } \quad T_{i} \geq 0
\end{array}
$$

We can form the Lagrange multiplier for this problem (see [27] for example) as:

$$
L\left(T_{1}, \ldots, T_{n}, \lambda\right)=\sum_{i=1}^{n} \sigma_{i}^{2} e^{-2 R_{i} T_{i}}+\lambda\left(\sum_{i=1}^{n} T_{i}-1\right) .
$$

Setting partial derivatives w.r.t $T_{i}(i=1 \ldots n)$ and $\lambda$ to zero and solving, we arrive at the solution:

$$
T_{i}= \begin{cases}\frac{\frac{\log \left(2 \sigma_{i}^{2} R_{i}\right)}{2 R_{i}}}{\sum_{i=1}^{n} \frac{\log \left(2 \sigma_{i}^{2} R_{i}\right)}{2 R_{i}}}, & R_{i}>\frac{1}{2 \sigma_{i}^{2}} \\ 0, & \text { otherwise }\end{cases}
$$

for $i=1 \ldots n$. The results of this optimization are presented in Figure 5 as a function of the transmit SNR at each buoy. Figure 6 presents the number of active buoys that the fusion center communicates with as a function of the tranmit SNR of the buoys. It is observed that for low transmit SNR's, the fusion center selectively communicates with only those nodes that have high source variances ( $\sigma_{i}^{2}$ as given in Table I) while at high transmit SNR's, it performs a form of water-filling (see [19] for example) across the buoys. We also note that hight source variances translate to more information content in the information theoretic sense and so the low SNR behaviour can also be interpreted as selectively communicating with the nodes that have more information to transmit that the others.

Let us assume that all the nodes have the same transmit SNR. As this SNR increases from zero, it is observed that the buoys are communicated with in the order of decreasing source variance - the buoy with the highest source variance (node 107 ) is turned on first while the one with the lowest source variance (node 101) is turned on last. When $\mathrm{SNR}_{0}$ is large, all nodes are allocated some time and no $T_{i}$ is zero. In this case, Equation (10) leads to $T_{i} \propto \frac{\log \left(2 \sigma_{i}^{2} R_{i}\right)}{R_{i}}$ and so, the time allocated to a particular buoy is directly proportional to the log of its observed variance and increases with its distance from the fusion center (since rate decreases as distance increases). It is then straightforward to see that buoy 107 always has the largest time allocated to it of all the buoys in the network (since it has the largest variance as well as distance from the fusion center) and is always the first node to be turned on for communication.

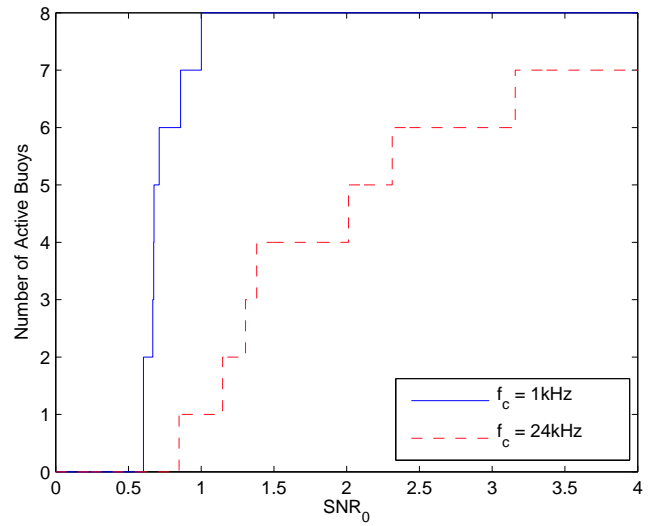

Fig. 6. The number of active buoys for the minimum sum distortion problem with a fixed fusion center for $f_{c}=1 \mathrm{kHz}$ and $f_{c}=24 \mathrm{kHz}$.

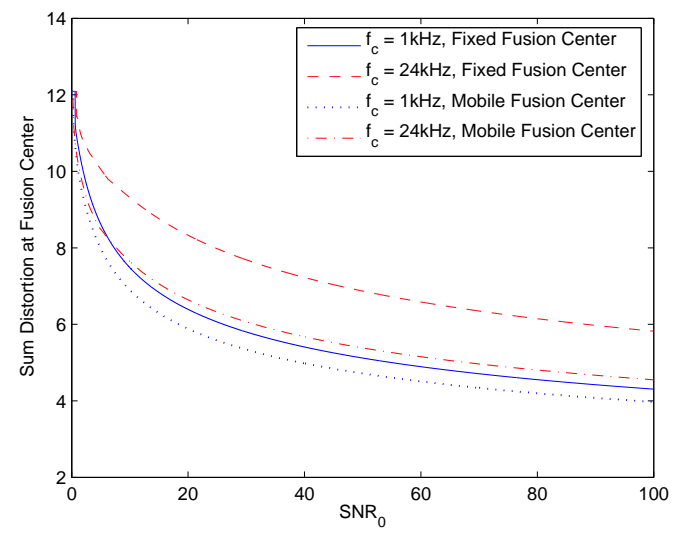

Fig. 7. The minimum sum distortion at the fusion center as a function of $\mathrm{SNR}_{0}$ optimized for the location of the fusion center for $f_{c}=1 \mathrm{kHz}$ and $f_{c}=$ $24 \mathrm{kHz}$. We also present the case when the fusion center is fixed.

\section{Problem Setup with a Mobile Fusion Center}

We now address the case when the fusion center is mobile - in effect, we now optimize the results of the last section as we vary the location of the base station from the fixed one assumed there. Some gains are immediately obvious - at low $\mathrm{SNR}_{0}$ 's, we would locate the fusion center at the node with the largest variance and turn all the other nodes off - thereby leading to some gains over the fixed base station approach. Figures 7 and 8 present the results of this optimization as a function of $\mathrm{SNR}_{0}$. We note that while there gains over the fixed base station scheme, they are not large when $f_{c}=1 \mathrm{kHz}-$ the gains are more striking when $f_{c}=24 \mathrm{kHz}$. We conjecture that this is because of the small distances involved in this network - the largest distance is on the order of hundreds of metres and for the attenuation coefficient implied by Equation (1), we do not have significant losses, when $f_{c}=1 \mathrm{kHz}$, over the underwater links for the communication losses to factor majorly into the problem. However, when $f_{c}=24 \mathrm{kHz}, \beta$ is significant enough so that moving the fusion center actually produces significant gains. 


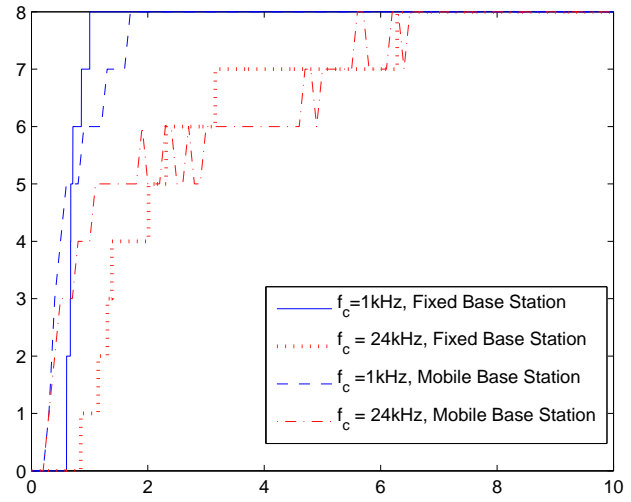

Fig. 8. The number of active buoys to minimize the sum distortion at the mobile fusion center as a function of $\mathrm{SNR}_{0}$. We also present the results when the fusion center is fixed.

\section{CONCLUSIONS}

We presented analytical and simulation results for the minimum sum distortion problem for data collected from a network of buoys. The analysis is presented for the cases when we have a fixed fusion center and the case when the fusion center is allowed to be mobile - thereby giving us additional parameters to optimize over. However, for the problem we set up, we note that the gains from the mobility of the fusion center, though noticable, are small. However, these results are particularly relevant for networks involving large distances and the case when most of the network is in inhospitable terrain and it is impractical to locate the fixed base station at a location of our choice.

There is plenty of scope for further work - the time and spatial correlations in the data collected at the buoys are currently ignored and can potentially be used to drive down distortions for a given rate constraint. This would lead to better results, but would involve more complicated processing at both the buoys and the fusion center. We also envision a future data collection activity to test the algorithms of the form described in this paper.

\section{ACKNOWLEDGEMENTS}

The data analyzed in this work was collected by the USC NAMOS team (J. Das, A. Pereira, B. Stauffer, D. Caron, A. Dhariwal, C. Oberg and G. Sukhatme). In addition, Dr. D. Liu is in the process of setting up an acoustic underwater testbed which will enable easier implementation and testing of algorithms of the form described in this work. The first author would also like to thank C. Choudhuri, S. Yerramalli and $\mathrm{G}$. Thatte for useful discussions.

\section{REFERENCES}

[1] D. Paley, F. Zhang, and N. Leonard, "Cooperative control for ocean sampling: The glider coordinated control system," IEEE Transactions on Control Systems Technology, vol. 16, no. 4, pp. 735-744, July 2008.

[2] F. Arrichiello, S. Chiaverini, and T. Fossen, "Formation control of marine surface vessels using the Null-Space-based Behavioral control," in Group Coordination and Cooperative Control, ser. K.Y. Pettersen, T. Gravdahl, and H. Nijmeijer (Eds.), Springer-Verlag's Lecture Notes in Control and Information Systems series, May 2006, pp. 1-19.
[3] E. Sozer, M. Stojanovic, and J. Proakis, "Underwater acoustic networks," IEEE Journal of Oceanic Engineering, vol. 25, no. 1, pp. 72-83, 2000.

[4] A. Dhariwal, B. Zhang, C. Oberg, B. Stauffer, A. Requicha, D. Caron, and G. S. Sukhatme, "Networked aquatic microbial observing system," in IEEE International Conference of Robotics and Automation (ICRA), Orlando, FL, USA, May 2006.

[5] G. S. Sukhatme, A. Dahriwal, B. Zhang, C. Oberg, B. Stauffer, and D. Caron, "The design and development of a wireless robotic networked aquatic microbial observing system," Environmental Engineering Science, vol. 24, no. 2, pp. 205-215, Oct 2007.

[6] B. Zhang and G. S. Sukhatme, "Adaptive sampling for estimating a scalar field using a mobile robot and a sensor network," in Proceedings of the IEEE International Conference on Robotics and Automation (ICRA), Roma, Italy, Apr 2007, pp. 3673-3680.

[7] S. Vedantam, W. Zhang, and U. Mitra, "A constrained channel coding approach to joint communication and channel estimation," IEEE Transactions on Information Theory, submitted Sept 2008.

[8] R. Willett, A. Martin, and R. Nowak, "Backcasting: Adaptive sampling for sensor networks," in Proceedings of the third international symposium on Information processing in sensor networks, 2004, pp. 124-133.

[9] A. Jain and E. Y. Chang, "Adaptive sampling for sensor networks," in the Proceedings of the First Workshop on Data Management for Sensor Networks (DMSN 2004), 2004.

[10] M. H. Rahimi, "Bioscope: Actuated sensor network for biological science," Ph.D. dissertation, Computer Science Department, Viterbi School of Engineering, USC, 2005.

[11] B. Zhang, M. Hansen, and G. Sukhatme, "Adaptive sampling for estimating a scalar field using a mobile robot and a sensor network," submitted to the IEEE Transactions on Robotics, 2008.

[12] A. Krause, C. Guestrin, A. Gupta, and J. Kleinberg, "Near-optimal sensor placements: Maximizing information while minimizing communiction cost," in Proccedings of the Fifth International Conference on Information Processing in Sensor Networks (IPSN'06), Apr 2006, pp. $2-10$.

[13] N. Roy, W. Burgard, D. Fox, and S. Thrun, "Coastal navigation - robot motion with uncertainty," in In AAAI Fall Symposium: Planning with POMDPs, 1998, pp. 35-40.

[14] R. Sim and N. Roy, "Global a-optimal robot exploration in SLAM," in the Proceedings of the IEEE/RSJ International Conference of Robotics and Automation (ICRA), 2005, pp. 661-666.

[15] B. Yamauchi, "A frontier-based approach for autonomous exploration," in the Proceedings of the IEEE International Conference of Robotics and Automation (ICRA), Jul 1997, pp. 146-151.

[16] E. M. Arkin, J. S. Mitchell, and G. Narasimhan, "Resource-constrained geometric network optimization," in Proceedings of the forteenth annual symposium on Computational geometry, 1998, pp. 307-316.

[17] A. Blum, S. Chawla, D. R. Karger, T. Lane, A. Meyerson, and M. Minkoff, "Approximation algorithms for orienteering and discountedreward tsp," in Proceedings of the 44th Annual IEEE Symposium on Foundations of Computer Science, 2003, pp. 46-55.

[18] H. L. V. Trees, Detection, Estimation, and Modulation Theory Part I. John Wiley and Sons, Inc., 1968.

[19] T. M. Cover and J. A. Thomas, Elements of Information Theory. John Wiley \& Sons, Inc., 1991.

[20] G. Thatte and U. Mitra, "Sensor selection and power allocation for distributed estimation in sensor networks: Beyond the star topology," IEEE Transactions on Signal Processing, July 2007, to appear.

[21] M. Stojanovic, "Underwater acoustic communications: Design considerations on the physical layer," Fifth Annual Conference on Wireless on Demand Network Systems and Services, pp. 1-10, 2008.

[22] J. Preisig, "Acoustic propagation considerations for underwater acoustic communications network development," SIGMOBILE Mob. Comput. Commun. Rev., vol. 11, no. 4, pp. 2-10, 2007.

[23] M. Stojanovic, "On the relationship between capacity and distance in an underwater acoustic communication channel,' in WUWNet '06: Proceedings of the 1st ACM international workshop on Underwater networks. ACM, 2006, pp. 41-47.

[24] I. L. L.M. Brekhovskikh, Yu. P. Lysanov, Fundamentals of Ocean Acoustics. Computer Sciences Corporation, Washington DC, USA: Springer, 2003.

[25] C. E. Shannon and W. Weaver, A Mathematical Theory of Communication. Champaign, IL, USA: University of Illinois Press, 1963.

[26] Y. Oohama, "The rate-distortion function for the quadratic Gaussian CEO problem," IEEE Transactions on Information Theory,, vol. 44, no. 3, pp. 1057-1070, May 1998.

[27] S. Boyd and L. Vandenberghe, Convex Optimization. Cambridge University Press, 2003. 